\title{
Alterstice
}

Revue internationale de la recherche interculturelle

International Journal of Intercultural Research

Revista International de la Investigacion Intercultural

\section{L'intégration des élèves nouveaux arrivants d'origine africaine dans les écoles de la division scolaire franco-manitobaine}

\section{Jacob Atangana-Abe et Mamadou Ka}

Volume 6, numéro 1, 2016

Prendre en compte la diversité à l'école

URI : https://id.erudit.org/iderudit/1038281ar

DOI : https://doi.org/10.7202/1038281ar

Aller au sommaire du numéro

Éditeur(s)

Alterstice

ISSN

1923-919X (numérique)

Découvrir la revue

Citer cet article

Atangana-Abe, J. \& Ka, M. (2016). L'intégration des élèves nouveaux arrivants d'origine africaine dans les écoles de la division scolaire franco-manitobaine. Alterstice, 6(1), 77-89. https://doi.org/10.7202/1038281ar
Résumé de l'article

Pour un pays comme le Canada, né de l'immigration et qui continue à se développer sur la base du multiculturalisme, l'intégration des nouveaux arrivants en milieu scolaire est un enjeu de taille. En effet, les écoles d'aujourd'hui sont composées de jeunes issus de milieux culturels et socioéconomiques divers. Au Manitoba en particulier, le nombre d'immigrants francophones s'est considérablement accru au sein de la communauté franco-manitobaine au cours des dix dernières années. Cela s'est traduit, au niveau des écoles de la Division scolaire franco-manitobaine (DSFM), par un accroissement de la population d'élèves nouveaux arrivants (ÉNA), population considérée à juste titre comme un enrichissement de cette communauté et de ses écoles, mais qui présente en même temps pour la division scolaire francophone des défis sur les plans de la pédagogie et de la communication, entre autres.

Basé sur une recherche empirique auprès des comités de parents des écoles de la DSFM, cet article porte sur le vécu des élèves nouveaux arrivants d'origine africaine dans ces écoles, avec l'objectif de comprendre comment elles font face à une telle réalité et de proposer des solutions pour une meilleure intégration de ces élèves. 


\title{
7
}

ARTICLE THÉMATIQUE

\section{L'intégration des élèves nouveaux arrivants d'origine africaine dans les écoles de la division scolaire franco-manitobaine}

\author{
Jacob Atangana-Abe ${ }^{1}$ et Mamadou Ka ${ }^{1}$
}

\begin{abstract}
Résumé
Pour un pays comme le Canada, né de l'immigration et qui continue à se développer sur la base du multiculturalisme, l'intégration des nouveaux arrivants en milieu scolaire est un enjeu de taille. En effet, les écoles d'aujourd'hui sont composées de jeunes issus de milieux culturels et socioéconomiques divers. Au Manitoba en particulier, le nombre d'immigrants francophones s'est considérablement accru au sein de la communauté francomanitobaine au cours des dix dernières années. Cela s'est traduit, au niveau des écoles de la Division scolaire franco-manitobaine (DSFM), par un accroissement de la population d'élèves nouveaux arrivants (ÉNA), population considérée à juste titre comme un enrichissement de cette communauté et de ses écoles, mais qui présente en même temps pour la division scolaire francophone des défis sur les plans de la pédagogie et de la communication, entre autres.

Basé sur une recherche empirique auprès des comités de parents des écoles de la DSFM, cet article porte sur le vécu des élèves nouveaux arrivants d'origine africaine dans ces écoles, avec l'objectif de comprendre comment elles font face à une telle réalité et de proposer des solutions pour une meilleure intégration de ces élèves.
\end{abstract}

\section{Rattachement des auteurs}

${ }^{1}$ École d’administration des affaires, Université de Saint-Boniface, Winnipeg, Canada

\section{Correspondance}

jatangan@ustboniface.ca

\section{Mots clés}

intégration scolaire, élèves nouveaux arrivants, Division scolaire franco-manitobaine

\section{Pour citer cet article}

Atangana-Abe, J. et Ka, M. (2016). L'intégration des élèves nouveaux arrivants d'origine africaine dans les écoles de la division scolaire franco-manitobaine. Alterstice, 6(1), 77-90. 


\section{Introduction}

L'immigration est devenue un facteur important de survie et de développement socioéconomique des communautés francophones en situation minoritaire. C'est pour cette raison que ces communautés développent, depuis quelques années, de nombreuses stratégies pour attirer et retenir des francophones venant de tous les coins du globe. Au niveau du gouvernement fédéral, un comité directeur Citoyenneté et Immigration Canada Communautés francophones en situation minoritaire a été mis sur pied en 2002. II a publié en 2003 un rapport intitulé Cadre stratégique pour favoriser l'immigration au sein des communautés francophones en situation minoritaire, dont les objectifs sont d'augmenter le nombre d'immigrants francophones dans les communautés francophones en situation minoritaire, de les accueillir et de les intégrer économiquement, socialement et culturellement. Ces initiatives gouvernementales et communautaires, pour n'en citer que quelques-unes, visent toutes à augmenter le nombre de francophones dans les provinces et territoires du pays. C'est ainsi qu'entre 2005 et 2014, le nombre annuel de résidents permanents venus au Manitoba est passé d'environ 8000 à plus de 16000 (Citoyenneté et Immigration Canada, 2015).

Au Manitoba, la Société franco-manitobaine (SFM), l'organisme porte-parole des francophones, a développé en octobre 2001 le projet "Agrandir l'espace francophone au Manitoba: toile de fond commune 2001-2050 ». Ce projet met en avant deux axes prioritaires pour l'agrandissement de la communauté francophone : la promotion du Manitoba français à l'échelle internationale et la révision des politiques fédérales et provinciales quant aux réfugiés, à la rétention des étudiants internationaux et au nombre d'immigrants francophones reçus dans ces communautés en comparaison avec le Québec (Therrien, 2002, p. 3).

La mise en œuvre de ces axes prioritaires a eu comme effet un accroissement du nombre de francophones dans la province (Ka, 2007). Ainsi, entre 2002 et 2013, le nombre d'immigrants francophones au Manitoba a plus que quadruplé, passant de 103 à 462 (Emploi et Immigration Manitoba, 2013). Pour les années à venir, sur les 20000 immigrants que le gouvernement provincial entend accueillir au Manitoba chaque année, la communauté franco-manitobaine cible $7 \%$ de nouveaux arrivants francophones par année.

Cette arrivée en grand nombre d'immigrants dans les communautés francophones du Manitoba va accentuer le défi du multiculturalisme non seulement au niveau de ces communautés, mais aussi au sein de la DSFM, qui voit déjà sa clientèle scolaire se diversifier sur les plans culturel, ethnique et racial. De fait, l'accueil des élèves nouveaux arrivants (ÉNA) dans les écoles de la DSFM est un phénomène soudain et récent (tableau 1).

Tableau 1 : Évolution des inscriptions des ÉNA dans les écoles de la DSFM

\begin{tabular}{lccc}
\hline \multicolumn{1}{c}{ Année scolaire } & Nombre d'élèves & Nombre d'écoles & Nombre de pays d'origine \\
\hline $2003-2006$ & 85 & 6 & 17 \\
$2006-2009$ & 227 & 11 & 35 \\
$2009-2012$ & 168 & - & 26 \\
$2012-2015$ & 135 & - & - \\
\hline
\end{tabular}

Source : Données fournies par la DSFM et compilées par les auteurs

En effet, en six ans, soit entre 2003 et 2009, la population des ÉNA dans les écoles de la DSFM a presque triplé, passant de 85 à 227, tandis que le nombre de pays de provenance est passé de 17 à 35 pour la même période. Étant entendu que le Manitoba vise à augmenter d'année en année le nombre de ses nouveaux arrivants, le nombre d’ÉNA inscrits dans les écoles de la DSFM va indubitablement augmenter dans les années à venir.

Les écoles de la DSFM, malheureusement, n'ont pas été préparées à faire face à cet afflux relativement soudain de ces ÉNA. Celles géographiquement proches de St-Boniface ont été les premières à y être confrontées, puis, d’année en année, ce phénomène s'est propagé à toutes les écoles urbaines de la DSFM, d'où la question de savoir comment cette réalité est vécue et prise en compte. 


\section{L'intégration des élèves nouveaux arrivants francophones en milieu scolaire au Canada}

Pour un pays comme le Canada, né de l'immigration et continuant à se développer sur la base du multiculturalisme, l'intégration des nouveaux arrivants dans les milieux scolaires restera toujours un enjeu de taille (Farmer, 2008; Robineau, 2010). Les écoles au sein des communautés francophones en situation minoritaire sont en effet de plus en plus composées de jeunes avec des habiletés langagières et culturelles variées et appartenant à des milieux socioéconomiques divers. Cet accroissement de la diversité ne va cependant pas de pair avec l'intérêt porté à ce sujet dans les recherches. Jacquet, Moore et Sabatier (2008) parlent de paucité de recherches sur la problématique de la gestion de la diversité dans les écoles en milieux linguistiques minoritaires au Canada en général et sur l'intégration des élèves issus de l'immigration en particulier.

Malgré cette paucité, des études récentes montrent qu'il existe de nombreux problèmes relatifs à l'intégration scolaire des élèves issus de l'immigration. Ces problèmes sont, pour la plupart, reliés à leur statut de double minorité et ont trait à la discrimination, aux défis d'adaptation à une nouvelle culture et à l'inadaptation aux méthodes d'apprentissage du pays d'accueil (Mc Andrew, Ledent et Ait-Said, 2006 ; Mc Andrew, Garnett, Ledent et Ungerleider, 2008 ; Murdoch et Ledent, 2010).

En milieu francophone minoritaire, la présence de jeunes immigrants dans les écoles francophones apparaît comme un parcours parsemé d'embûches, où il faut non seulement affronter un nouveau système et une nouvelle culture scolaires, se faire de nouveaux amis et apprendre l'anglais, mais aussi se familiariser avec les variétés du français canadien, voire apprendre le français standard (Émond, 2008 ; Moke Ngala, 2005). Pour un grand nombre de jeunes nouveaux arrivants, franchir ces différents obstacles devient un parcours du combattant dans la mesure où l'expérience qu'ils ont vécue dans leur pays d'origine semble souvent peu pertinente pour leur nouvelle situation. Leur vie se trouve souvent d'autant plus bouleversée que la plupart des écoles francophones en milieu minoritaire et son personnel ne sont pas du tout préparés à les recevoir et à leur apporter l'aide dont ils ont besoin (Gagnon, 2007 ; Jacquet, Moore et Sabatier, 2008 ; Moke Ngala, 2005). En effet, un certain nombre d'auteurs constatent, pour le déplorer, que dans les provinces où les francophones sont en situation minoritaire, il existe dans les écoles anglophones plusieurs outils d'intégration des élèves nouveaux arrivants alors que dans les écoles francophones, ces outils sont mis en place de façon ponctuelle et embryonnaire (Carlson Berg, 2010 ; Gallant et Denis, 2008 ; Gérin-Lajoie et Jacquet, 2008).

Face à ces défis d'intégration, quelques pistes de solution ont été proposées dont certaines sont déjà mises en œuvre. C'est le cas de l'idée selon laquelle une formation sur la diversité culturelle devrait être offerte aux enseignants afin de les aider à tenir compte de cette incontournable réalité dans leur enseignement (Émond, 2008 ; Gallant et Denis, 2008). Dans la même veine, Bouchamma (2008) recommande une collaboration accrue entre l'école et les familles afin d'établir un dialogue et de soutenir la réussite des jeunes. De même Émond (2008) souligne par ailleurs l'importance d'une représentation des communautés nouvellement arrivées dans les écoles de langue française, tant au niveau du corps enseignant qu'au sein des comités de parents.

Dans le registre de ce qui est concrètement fait dans les écoles francophones en situation minoritaire, Gérin-Lajoie et Jacquet (2008) notent qu'en Ontario et en Colombie-Britannique, les écoles francophones célèbrent la diversité à travers le port vestimentaire et la musique. Pour Farmer et Labrie (2008), les stratégies d'inclusion des élèves nouveaux arrivants sont actualisées à travers trois dispositifs : la mise en place d'un comité d'admission des élèves immigrés, des programmes de perfectionnement du français et un programme d'accueil qui vise à faciliter l'insertion sociale des jeunes en général. Robineau (2010) a recensé d'autres expériences qui ont été explorées à travers le Canada, dont l'élaboration d'une trousse d'accompagnement des élèves immigrés au NouveauBrunswick, l'embauche d'un personnel scolaire aux origines variées dans certaines écoles en Ontario et la création, dans la même province, d'un site web pour les nouveaux arrivants ou encore la mise en place d'un comité directeur des services d'accueil et d'éducation pour la communauté immigrante du Centre-Nord de l'Alberta.

Pour ce qui est du Manitoba spécifiquement, le nombre d'immigrants francophones dans cette province s'est considérablement accru au cours des dix dernières années (Emploi et Immigration Manitoba, 2013). Cet accroissement est dû à un afflux de réfugiés francophones venant des pays où l'éducation est en situation de crise 
(Ka, 2007 ; Piquemal et Bolivar, 2009 ; Piquemal et Murenzi, 2009 ; Piquemal, Bolivar et Bahi, 2009). Certains jeunes élèves ont de ce fait beaucoup de problèmes d'intégration dans les écoles de la DSFM à cause des traumatismes psychologiques liés à des expériences pré-migratoires (Jacquet, Moore, Sabatier et Masinda, 2008). D'autres ont des difficultés d'intégration socioéconomique (Mackay et Tavares, 2005 ; McBrien, 2005) « en raison de leur phénotype, de leur ethnicité et de leurs croyances islamiques, tous des éléments qui, selon les études réalisées antérieurement, alimentent la discrimination et le racisme à leur égard "(Kanu, 2009, p. 126). Ces difficultés, comme il vient d'être souligné, sont particulièrement prégnantes à l'école francophone qui, souvent, n'a pas les ressources matérielles et humaines pour faciliter la vie de ces jeunes immigrants en milieu scolaire minoritaire. Ces difficultés d'intégration sont par ailleurs, de notre point de vue, accentuées par le fait d'une insuffisance d'études ou de recherches portant sur ce sujet à travers le Canada. En effet, les différentes expériences d'inclusion des ÉNA recensées çà et là, pour louables qu'elles soient, restent malheureusement parcellaires et ne s'intègrent pas à une stratégie ou une vision globale. Au Manitoba, ces expériences sont peu documentées et la présence des ÉNA dans les écoles de la DSFM reste encore un phénomène à explorer. C'est ce que tente de faire cette recherche, qui vise un double objectif : comprendre la réalité des ÉNA d'origine africaine dans les écoles de la DSFM et proposer des solutions pour leur meilleure intégration dans ces écoles.

\section{Méthodologie}

Cette recherche, qui se situe dans une perspective de compréhension des défis d'intégration des élèves nouveaux arrivants d'origine africaine dans les écoles de la DSFM, repose sur une approche qualitative. Comme l'affirment Taylor et Bogdan (1984), ce type de recherche produit et analyse des données descriptives, telles que les paroles écrites ou dites et le comportement observatoire des personnes. Elle vise le sens et l'observation d'un phénomène social en milieu naturel. Dans cette quête de compréhension, l'outil de recherche retenu ici est l'entretien de groupe, sur lequel nous reviendrons après avoir présenté une définition de l'élève nouvel arrivant et nos objectifs.

La DSFM considère comme nouvel arrivant tout élève venant de l'extérieur du Canada et inscrit dans l'un de ses établissements scolaires depuis moins de cinq ans (Division scolaire franco-manitobaine, 2008). Les nouveaux arrivants qui choisissent les écoles de la DSFM sont d'horizons divers ${ }^{1}$, mais la très grande majorité est composée de réfugiés d'origine africaine, une catégorie qui présente des défis quant à son intégration dans les écoles de la division scolaire, d'où l'intérêt de cette étude, dont l'objectif est de comprendre la réalité de leur présence. Pour atteindre cet objectif, nous avons utilisé des entretiens de groupe, outil qui permet non seulement de collecter des informations sur un sujet ciblé, mais aussi d'explorer les préoccupations particulières des participants (Malhotra, 2011). Bien plus, les entretiens de groupe encouragent l'interaction entre participants, ce qui permet de stimuler différents points de vue grâce à la discussion.

Dans le cadre de cette étude, les entrevues de groupe ont duré en moyenne une heure. Elles ont été limitées aux comités de parents des écoles de la DSFM de la ville de Winnipeg (Manitoba), où l'on note une forte présence d'ÉNA. Les comités de parents ont été choisis comme base d'entretien du fait de leur composition : ils se présentent en effet comme un concentré des parties prenantes d'une école: parents (de toutes origines), membres de la direction de l'école, orthopédagogues, enseignants, etc. Le nombre de parents dans les comités varie selon les écoles, et il faut souligner que les parents immigrants sont très peu représentés dans les comités. Le tableau 2 donne quelques indications sur ces rencontres, qui s'articulaient autour des questions suivantes: comment l'école a-t-elle vécu l'arrivée des ÉNA dans ses effectifs ? Quels sont les défis que pose cette arrivée à l'école? Comment s'est faite l'adaptation des ÉNA au sein de l'école? Quels sont les outils, politiques et programmes qui ont été mis en place pour faciliter l'intégration des ÉNA?

Les rencontres ont été organisées les soirs et coïncidaient généralement avec une réunion régulière du comité de parents. Lors des entretiens, nous étions deux chercheurs avec des rôles bien définis, l'un prenant les notes, l'autre animant la rencontre.

\footnotetext{
${ }^{1}$ Afrique du Sud, Belgique, Bénin, Brésil, Bulgarie, Burundi, Cameroun, Chili, Congo Brazzaville, République démocratique du Congo, Côte d'Ivoire, Djibouti, Égypte, Éthiopie, France, Gabon, Ghana, Guinée, Haïti, Île Maurice Iran, Kenya, Liban, Mali, Maroc, Mauritanie, Mozambique, Nigeria, Ouganda, Russie, Rwanda, Sénégal, Somalie, Suisse, Tanzanie, Tchad, Togo, Tunisie, Zambie, Zimbabwe,
} 
Tableau 2 : Liste des comités des parents rencontrés

\begin{tabular}{cc}
\hline Comité des parents & Nombre de participants \\
\hline École A & 7 \\
École B & 8 \\
École C & 7 \\
École D & 7 \\
École E & 11 \\
École F & 10 \\
Collège X & 11 \\
\hline Total & 61 \\
\hline
\end{tabular}

\section{Résultats}

De l'analyse de contenu des entrevues, il ressort deux grands thèmes relatifs aux défis que représentent les ÉNA pour les écoles et aux stratégies développées par les écoles face à la présence des ÉNA.

\section{Les défis que représentent les ÉNA pour les écoles de la DSFM}

De prime abord, tout le monde s'entend pour reconnaître que les élèves nouveaux arrivants représentent une richesse pour les écoles de la DSFM.

La présence des ÉNA enrichit notre établissement... c'est du beau monde qui nous arrive. (Une responsable d'école)

C'est très positif [la présence des ÉNA] pour l'école, car ça nous force à revoir nos façons de faire. On est obligé de revoir nos tournures de phrases. (Une enseignante)

Cependant, il n'en demeure pas moins vrai que la présence des ÉNA présente aussi des défis importants, vécus comme des entraves à l'intégration et à la réussite scolaire. Ces défis sont d'ordre économique, culturel et pédagogique.

\section{Les défis d'ordre économique}

Les élèves nouveaux arrivants, dont la majorité est composée de réfugiés, subissent la précarité économique à laquelle leurs parents sont confrontés. Cette précarité se ressent dans leur scolarité, par la difficulté des parents à subvenir aux besoins relatifs à la vie scolaire et parascolaire. Cette difficulté est de nature à compromettre la participation des enfants à la vie de l'établissement qu'ils fréquentent. Les personnes rencontrées nous ont fait part du fait que certains élèves étaient incapables de payer les frais exigés pour participer à un camp d'hiver ou à une sortie à caractère culturel (visites de musées, assistance à une pièce de théâtre, etc.). Certains élèves viennent aussi à l'école le ventre vide, sans avoir pris de petit déjeuner. D'autres ne parviennent pas à s'habiller convenablement l'hiver. II arrive dans certains cas que, faute de changer d'habits ou de les laver, certains élèves en viennent à indisposer leurs camarades par des odeurs ou des habits sales. Ces facteurs seraient propices au rejet et à la marginalisation des ÉNA dans les écoles et représenteraient des obstacles à l'intégration de ces élèves, ce qu'ont souligné Murdoch et Ledent (2010).

\section{Les défis d'ordre culturel}

Dans les entretiens, il a été mentionné que certains jeunes ÉNA d'origine africaine ont tendance à prendre possession d'un crayon ou de tout autre article qui traîne sans se soucier de savoir à qui il appartient, acte qui peut être perçu comme du vol dans le contexte culturel canadien en général, et manitobain en particulier. Il a aussi été signalé que certains ÉNA d'origine africaine, lors de la période des repas, avaient tendance soit à manger en groupe dans une même assiette, soit à se servir sans demander la permission au propriétaire du repas. 
Les deux comportements ci-dessus, qui apparaissent à tout le moins incongrus dans un contexte canadien, sont plutôt courants dans une école africaine, où beaucoup d'élèves s'échangent du matériel scolaire (livres, crayons, calculatrices, gommes à effacer, etc.) et où manger tout seul dans son propre plat est perçu comme un acte égoïste. Ces situations relèvent de cas d'acculturation de l'ÉNA par rapport à son nouveau milieu d'adoption, acculturation qui se manifesterait par ce qui est communément appelé des "faux pas culturels » (Fournier, 2013). ॥ s'agit de gestes, de comportements ou d'attitudes mal interprétés par les membres de la communauté d'accueil, qui empêchent l'intégration harmonieuse de l'ÉNA et créent chez lui un sentiment de frustration ou de rejet.

Par ailleurs, plusieurs enseignants interrogés ont mentionné que certains ÉNA manquent de compétences linguistiques en français, en anglais, et parfois dans les deux langues. D'après ces derniers, ce handicap complique la communication au sein des groupes et limite la participation des ÉNA aux activités pédagogiques et sociales. D'autres s'expriment dans ces langues avec un accent. La communication étant à double sens, il arrive tout aussi souvent que l'ÉNA ne comprenne pas l’accent franco-manitobain.

Socialement, les ÉNA sont acceptés. Cependant, il y a un problème de langue. [Certains] ont un accent prononcé. C'est très frustrant pour eux aussi. (Une enseignante)

Est-ce un trait culturel que certains ÉNA d'origine africaine tendent à se faire justice ou à régler leurs conflits à coups de poing ? Difficile à dire dans la mesure où ce comportement, assez fréquent chez les réfugiés par exemple, serait la conséquence du parcours de ces élèves. En effet, l'on sait que la vie dans un camp de réfugiés n'est pas un long fleuve tranquille et que le recours à la force, dans ce milieu-là, est un moyen de régulation sociale très usité.

Il y a enfin le fait que les ÉNA, de façon générale, ne sont pas encore habitués au langage politiquement correct canadien et à la signification de certains gestes ou comportements. Ainsi, l'usage des mots "grosse ", " grasse ", "épais ", etc. n'a pas la même signification chez les ÉNA que chez les Canadiens non immigrants. De même, les contacts physiques fréquents seraient une marque de proximité et d'amitié chez les ÉNA d'origine africaine, alors que chez leurs camarades franco-manitobains ces contacts peuvent avoir une tout autre connotation.

\section{Les défis pédagogiques}

Les défis pédagogiques diffèrent selon qu'il s'agit des ÉNA réfugiés ou des ÉNA immigrants indépendants. Dans le cas des réfugiés, leur parcours scolaire a été marqué, dans les régions d’où ils viennent, par une scolarité constamment interrompue voire inexistante, scolarité qualifiée par l'Unesco d' « éducation en situation de crise ».

28 millions d'enfants sont privés d'éducation en raison des conflits armés qui les exposent aux viols, aux violences sexuelles, à des attaques ciblées sur leurs écoles et à d'autres atteintes aux droits de l'homme. (Unesco, 2011)

Avant d'arriver au Manitoba, beaucoup d'élèves avec un statut de réfugié ont vécu des traumatismes et des expériences horribles dont le souvenir les habite toujours (assassinat de membres de leur famille, mutilations de bras ou de pieds, viols, etc.). Faute de traitement médical ou psychologique, ces expériences ont une influence négative sur leur apprentissage.

La communauté franco-manitobaine accueille de nombreuses familles ayant vécu ce type de situations. Environ $45 \%$ de toute la clientèle de l'Accueil francophone provient de la République démocratique du Congo, pays dévasté par la guerre. Concernant cette catégorie, une enseignante nous a raconté comment, lors de la visite au Festival du voyageur, quelques ÉNA de sa classe ont été terrifiés d'entendre des coups de fusil. De même, lors de la célébration de la fête du Canada, toute une famille de réfugiés s'était cachée sous le lit en entendant le bruit des feux d'artifice. Dans l'un et l'autre cas, les coups de feu réveillaient chez le eux le souvenir de l'état de guerre qui les avait fait partir de leur pays d'origine.

Pour les autres ÉNA, nos entrevues ont révélé que ceux qui arrivent au Canada avec un niveau scolaire en deçà de celui de leurs camarades canadiens du même âge sont, malgré tout, intégrés dans les mêmes classes que leurs congénères canadiens. Certains de ces ÉNA se retrouvent ainsi en $6^{\mathrm{e}}$ année du fait de leur âge, alors qu’ils n’ont en 
réalité que le niveau de $3^{\mathrm{e}}$ ou de $4^{\mathrm{e}}$ année. De plus, il arrive que certains ÉNA intègrent une classe plusieurs mois après la rentrée officielle. On saisit aisément les répercussions néfastes d'une telle situation sur le plan pédagogique tant pour l'élève que pour le reste de la classe et l'enseignante, si des mesures de soutien efficaces ne sont pas disponibles.

Enfin, beaucoup d'enseignants notent que, toutes catégories confondues, l'ignorance du contexte canadien d'une part et les différences d'approches pédagogiques entre ce qui se fait en Afrique et ce qui se fait au Canada d'autre part peuvent constituer des handicaps à l'apprentissage de certains ÉNA. En classe, la plupart des exemples et des exercices étant tirés du contexte canadien, Les ÉNA, qui ont vécu dans un contexte culturel différent, éprouvent parfois des difficultés de représentation des faits tirés de cet environnement.

J'ai deux élèves qui sont arrivées au mois de janvier, puis en février, elles étaient chez nous... j'ai fait un test en classe sur le ski de fond. Elles ne comprenaient pas. Alors, je leur ai dit de laisser tomber (Une enseignante)

Les différences d'approches pédagogiques entre l'Afrique et le Canada constituent aussi un défi pédagogique à la fois pour les ÉNA et les enseignants. Au Canada, l'accent est mis sur l'expérimentation, alors qu'en Afrique c'est la mémorisation qui est souvent valorisée. D'après une enseignante, ces différences au niveau de l'approche pédagogique sont perceptibles en mathématiques:

Les maths sont enseignées différemment [en Afrique et au Canada]. Les ÉNA semblent être habitués aux algorithmes alors qu'ici on met l'accent sur le processus et non sur la réponse. (une enseignante)

Au-delà de ces facteurs liés au vécu des nouveaux arrivants avant leur arrivée au Manitoba, plusieurs enseignantes affirment que le principal défi de l'ÉNA est au niveau du langage.

Certains [ÉNA] vous arrivent en $6^{\mathrm{e}}$ année, mais ils ne parlent pas français... J'ai eu des difficultés avec un qui n'arrive pas à s'exprimer. (Une enseignante)

Autant de situations qui entraînent de mauvais rendements scolaires, accroissent la frustration et augmentent le risque de décrochage de l'élève. Les parents des autres élèves issus de la communauté d'accueil expriment des préoccupations quant au fait que le temps passé par l'enseignante pour essayer de relever le niveau des ÉNA serait pris dans le temps «normal » d'enseignement, ce qui pénaliserait les autres élèves. Les enseignantes quant à elles ont regretté que la présence des ÉNA demande des ressources supplémentaires qu'elle n'ont pas, et que ne compense pas totalement la présence d'un ou une auxiliaire, l'une des stratégies développées par les écoles pour faire face aux défis posés par la présence de ces élèves.

\section{Les stratégies développées par les écoles}

De l'analyse de contenu des entrevues, on peut dégager trois types de stratégies développées par les écoles de la DSFM face à la présence des ÉNA : les stratégies axées sur les élèves eux-mêmes, les stratégies développées à l'égard de leurs enseignants et les stratégies en direction de leurs parents.

\section{Les stratégies axées sur les ÉNA eux-mêmes}

Ces stratégies, qui se rapportent à l'institution de cours de rattrapage et au soutien matériel aux ÉNA qui sont dans le besoin, visent à faciliter l'apprentissage de ces derniers et à les amener à s'intégrer au milieu scolaire.

\section{- L'institution de cours de rattrapage}

Au vu du parcours scolaire chaotique de certains élèves avant leur arrivée au Canada, les écoles ont pour la plupart mis en place un processus d'évaluation préalable des ÉNA au moment de l'inscription.

On évalue les compétences pour voir si on place l'ÉNA dans une classe de rattrapage. On évite la ségrégation, car c’est important de les garder motivés. (Un membre de la direction de l'école X) 
Les cours de rattrapage se présentent comme la conséquence logique de l'évaluation préalable dont nous venons de parler. Ils ont donc pour objectif la mise à niveau des élèves dans les matières où cette évaluation a révélé des insuffisances. À l'école $X$, les cours de rattrapage concernent surtout l'informatique, les sciences humaines, les mathématiques et l'anglais. Cet établissement a aussi mis en place un cours d'initiation aux métiers destiné aux élèves de la $9^{\mathrm{e}}$ à la $12^{\mathrm{e}}$ année, pour offrir les rudiments d'une formation professionnelle à ceux et celles qui, pour une raison ou une autre, ne pourraient pas s'engager dans des études post-secondaires.

- Le soutien matériel aux ÉNA dans le besoin

Chaque école de la DSFM essaie à son niveau d'éviter que les conditions économiques des enfants n'affectent négativement leur parcours scolaire en général, ÉNA comme franco-manitobains de souche. Le soutien matériel aux élèves relève du projet que l'argent ne soit pas un handicap pour l'intégration scolaire de l'élève. Ainsi, une fois par mois, l'école $Y$ vend de la pizza, et ces revenus aident à alimenter un fonds de soutien aux élèves démunis. L'école $Y$, en partenariat avec le Centre $Z$ (organisme de charité qui distribue gratuitement des vêtements aux personnes démunies), fournit quelquefois des manteaux d'hiver et des habits aux élèves qui ne peuvent se les procurer par leurs propres moyens.

L'école X est celle qui a poussé plus loin l’assistance matérielle aux élèves démunis :

On veut rendre leur expérience à l'école secondaire remplie [...] On veut qu'ils soient des élèves comme tout le monde. (Personnel de direction)

Grâce à un octroi du gouvernement, cet établissement fournit notamment aux élèves dans le besoin des billets d'autobus, des effets scolaires et des vêtements (manteaux d'hiver notamment dans les cas extrêmes). Cet octroi sert aussi à couvrir le coût des cotisations et des activités scolaires et sportives, et favorise de ce fait la participation des élèves à la vie scolaire.

Affiliée aux clubs des petits déjeuners du Canada (CPDC), un partenariat qui lui permet de recevoir de la part des CPDC une subvention annuelle, des denrées alimentaires et des ustensiles de cuisine, l'école $X$ offre également des petits déjeuners à ceux de ses élèves qui ne peuvent s'en offrir.

Dans les autres établissements, les petits déjeuners sont financés par les comités des parents. C'est le cas à l'école $Z$, où environ $30 \%$ des élèves en bénéficient et où le concours du comité de parents est d'environ 2000 dollars. Toujours à l'école Z, il existe un fonds d'inclusion, financé lui aussi par les activités du comité de parents, qui sert à aider les enfants dans le besoin. Malheureusement, ce fonds demeure insuffisant.

Comme vous le savez, la pauvreté enfantine augmente ce qui fait que le fonds est insuffisant, car il subventionne de nombreuses familles. (un parent d'élève)

Les stratégies développées par les écoles à l'égard des enseignants

Comme nous l'avons relevé plus haut, les enseignants des écoles de la DSFM n'ont été préparés, ni lors de leur formation générale ${ }^{2}$ ni dans leur parcours professionnel, à faire face aux défis induits par la présence des ÉNA. Conscientes de cet état de fait, les écoles ont mis à la disposition des enseignants un certain nombre de ressources, qui ont trait soit à l'adjonction d'auxiliaires soit à l'offre de programmes de renforcement des capacités interculturelles.

C'est la présence des ÉNA avec des besoins de formation spécifiques qui a amené les écoles à recruter des auxiliaires, c'est-à-dire des personnes embauchées pour appuyer le travail des enseignantes permanentes par rapport aux élèves qui ont des besoins spéciaux en termes de rattrapage scolaire. L'importance des auxiliaires dans les écoles a été unanimement reconnue. Cependant, leur contribution reste en deçà des attentes des écoles, du fait

${ }^{2}$ II y a moins de cinq ans qu'un cours sur la diversité culturelle dans les écoles a été mis sur pied à la Faculté d'éducation de I'Université de Saint-Boniface. 
qu'ils sont en nombre insuffisant et qu'ils ont, pour la plupart, une formation générale qui ne leur permet pas de répondre de façon professionnelle aux besoins spécifiques de la clientèle. Tirer le meilleur parti des auxiliaires nécessite de l'argent (pour un recrutement supplémentaire ou un complément de formation), ce que les écoles n'ont malheureusement pas.

Les écoles ont aussi conscience de la nécessité de doter leur personnel d'habiletés interculturelles pour leur permettre de mieux répondre aux besoins des ÉNA. Elles encouragent donc leur personnel à suivre des séminaires de formation dans ce sens. Ainsi, un séminaire destiné aux personnels des écoles de la DSFM a été spécialement développé par l'Accueil francophone sur la compréhension de la culture africaine et sur le développement des habiletés de communication avec les Africains.

\section{Les stratégies développées par les écoles en direction des parents}

Les écoles souhaitent voir les parents des ÉNA d’origine africaine s'impliquer davantage dans le suivi pédagogique de leurs enfants d'une part et dans les activités parascolaires d'autre part. Malheureusement, les parents des ÉNA brillent par leur absence, aussi bien dans la plupart des comités scolaires que dans les activités parascolaires.

La principale stratégie développée par les écoles à l'égard des parents des ÉNA consiste à garder un contact permanent avec ceux-ci, à les informer sur les performances de leurs enfants et à les mettre au courant des activités parascolaires qui sont programmées.

Malheureusement, la communication avec les parents est souvent tributaire d'obstacles inhérents à toute communication interculturelle. Certains malentendus apparaissent donc, rendant les écoles plus circonspectes. Ainsi, lorsque l'école conseille une évaluation psychologique d'un ÉNA, certains parents en déduisent que leur enfant est atteint de folie (perçue comme une honte pour la famille). Par ailleurs, certains enseignants ont peur que leur communication aux parents, au lieu d'aider l'élève, ne lui porte préjudice.

II faut savoir quoi dire aux parents pour éviter des châtiments corporels à l'enfant [...] II y en a eu une fois et on en a parlé aux parents. Je ne suis pas sûre qu'ils ont compris. (Une enseignante)

Même si elles ont été développées au fur et à mesure de l'apparition des défis et même si beaucoup reste à faire, il faut reconnaître que les stratégies développées par les écoles sont assez efficaces. Cependant, il est à regretter qu'elles restent cloisonnées et fragmentaires. Chaque école essaie ainsi de réinventer la roue, car il existe très peu d'échanges et de partage d'expériences entre écoles. De plus, les stratégies mises en place ne s'intègrent pas dans un modèle global, qui reste à inventer.

\section{Conclusion}

Cette étude visait à comprendre la réalité de la présence des ÉNA d'origine africaine dans les écoles de la DSFM. Nos résultats mettent en exergue les défis auxquels font face à la fois ces élèves nouveaux arrivants, les écoles et les parents. Par exemple, la précarité économique des parents peut se révéler un obstacle à l'intégration des ÉNA, car ceux-ci ont de la difficulté à défrayer les frais pour certaines activités scolaires. Ces élèves connaissent aussi des problèmes d'acculturation, car très souvent leur culture ou leurs habitudes diffèrent considérablement de celles du pays d'accueil. Nos résultats soulignent aussi les défis pédagogiques auxquels font face certains jeunes immigrants qui fréquentent les écoles de la DSFM : ces jeunes viennent pour la plupart de pays où les méthodes pédagogiques auxquelles ils ont été habitués sont différentes de celles développées au Canada. Alnsi, dans le cas des réfugiés, notre étude a fait ressortir que les difficultés d'intégration sont liées à des traumatismes vécus dans le pays d'origine.

L'analyse des données montre que, face aux défis ci-dessus, diverses stratégies axées sur les ÉNA, les enseignants et les parents ont été développées par les écoles de la DSFM pour faciliter l'intégration de ces jeunes immigrants. Ainsi, des cours de rattrapage sont dispensés et un soutien matériel est fourni aux ÉNA pour faciliter leur apprentissage, et des sessions de formation pour renforcer les capacités interculturelles sont dorénavant offertes 
au personnel enseignant et au personnel administratif. Les parents, quant à eux, sont encouragés à s'impliquer davantage dans l'éducation scolaire de leurs enfants.

Cependant, ces stratégies, pour pertinentes qu'elles soient, seraient beaucoup plus efficaces si elles s'inséraient dans un réseau de concertation plus étroite entre l'école, les parents et la communauté. C'est ce qui nous amène à proposer un modèle global de concertation intégrative des ÉNA, qui consiste à amener les parents, la communauté et l'école à entreprendre un dialogue permanent (figure 1). Elle fait appel à un ensemble de discussions sur les mesures à prendre pour faciliter l'intégration scolaire des ÉNA et repose sur une triple base : la concertation parents-école, la concertation parents-communauté et la concertation communauté-école (figure 1).

\section{Figure 1 : Le modèle global de concertation intégrative des ÉNA}

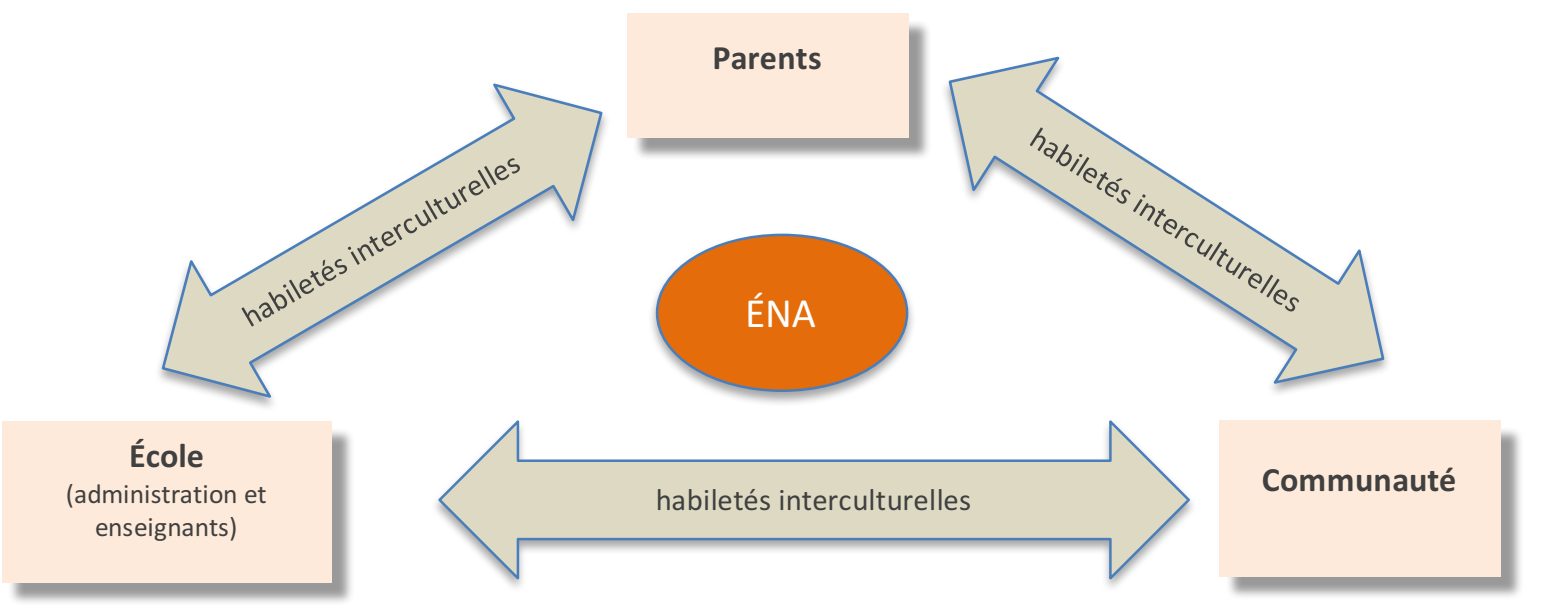

La concertation a souvent été présentée comme une condition sine qua non de la réussite scolaire des élèves (Bilodeau, Lefebvre, Bélanger, Deshaies et Gagnon, 2010 ; Deslandes, 2010a ; Hohlfield, Ritzhaupt et Barron, 2010). Cependant, dans un contexte multiculturel comme c'est le cas ici, elle se réduirait à un langage de sourds si elle n'était pas stimulée par la maîtrise des habiletés interculturelles par chacun des intervenants.

\section{Concertation parents-école}

Dans les écoles de la DSFM, la concertation avec les parents des ÉNA d'origine africaine est, comme nous l'avons vu, encore nimbée de multiples incompréhensions : les écoles déplorent la faible implication des ÉNA à l'éducation formelle de leurs enfants, tandis que les parents, de leur côté, ne comprennent pas tout à fait le rôle que veut leur faire jouer l'école. Pourtant, malgré ces incompréhensions, il faut reconnaître que la collaboration entre les parents immigrants et l'école, c'est-à-dire l'administration et les enseignants, comporte de nombreux avantages, aussi bien pour les enseignants et l'administration scolaire que pour les parents, car elle permet d'établir un pont entre ces deux parties qui interviennent dans la vie de l'enfant (Benoit, Rousseau, Ngirumpatse et Lacroix., 2008). La collaboration parents-école aurait donc le potentiel d'aider les élèves à obtenir de meilleurs résultats aux tests, à avoir un bon moral et un bon comportement et une attitude positive vis-à-vis de l'école. Elle permettrait de créer un dialogue et une complicité entre l'école et les familles et une implication de ces dernières dans l'éducation de leurs enfants (Deslandes, 2010b ; Farmer, 2010 ; Vatz Laaroussi, Kanouté et Rachédi, 2008).

\section{Concertation parents-communauté}

La communauté est définie ici comme le milieu de vie des élèves et des parents, qui comprend entre autres l'école, les élèves et les organismes communautaires (Kanouté, André, Charette, Lafortune, Lavoie et Gosselin-Gagné, 2011). 
Généralement, les organismes communautaires d'accueil sont le premier point de contact avec les nouveaux arrivants. Le dialogue permanent entre les parents immigrants et la communauté s'avère donc important, et ce, d'autant plus que la communauté regroupe de nombreux organismes appelés à intervenir, de l'accueil jusqu'à l'intégration économique, sociale et scolaire des nouveaux arrivants. Dans ce cadre, de nombreux services et activités (camps d'été, cours d'alphabétisation, cours de rattrapage scolaire, etc.) destinés aux immigrants ont été mis sur pied pour faciliter l'intégration. Le dialogue permet à ces derniers d'être au courant des ressources disponibles dans la communauté et d'en profiter pour devenir partie prenante de l'éducation formelle de leurs enfants.

\section{Concertation communauté-école}

L'école et la communauté doivent développer un dialogue permanent pour faciliter l'intégration des ÉNA de manière générale. Ainsi, l'école peut faire exploiter à bon escient les services dont disposent les organismes d'accueil des nouveaux arrivants. Au Manitoba, quoique encore timidement, certaines écoles de la DSFM profitent des ateliers de sensibilisation et des services d'interprétation offerts par l'Accueil francophone pour améliorer les compétences interculturelles de leurs enseignants.

En définitive, il faut rappeler que toutes les concertations à développer doivent être centrées sur l'ÉNA, pour faciliter son processus d'intégration scolaire (Benimmas et Kamano, 2009). Elles doivent aussi être sous-tendues par la maîtrise par les uns et les autres d'habiletés interculturelles.

\section{Habiletés interculturelles}

La concertation ne peut être efficace que si tous les partenaires acquièrent suffisamment d'habiletés interculturelles pour être en mesure de communiquer et de se comprendre. L'acquisition de ces habiletés passe par exemple par le développement de sessions de formation axées sur la communication interculturelle. Ces formations ont pour objectif de sensibiliser les uns et les autres à la notion de culture et à celle de diversité culturelle et de les aider à acquérir des connaissances interculturelles facilitant le dialogue et la concertation. Dans le contexte de diversité culturelle qui est le nôtre aujourd'hui, tout enseignant, pour être véritablement efficace dans sa salle de classe, doit être culturellement compétent.

Si nous pensons que la concertation entre les parties prenantes et la maîtrise des habiletés interculturelles par chacune d'elles est une condition essentielle à l'intégration des ÉNA dans les écoles en général, il reste que les données sur lesquelles cette étude s'appuie ont été obtenues auprès d'une seule division scolaire (la DSFM) et ne concernent que les ÉNA d’origine africaine. La validité externe de nos résultats et celle de la principale recommandation que nous formulons reste donc encore à confirmer. Aussi les recherches futures devraient-elles s'étendre à tous les ÉNA, quelle que soit leur origine, et aux divisions scolaires francophones des autres provinces. Et quid des écoles d'immersion française, dont la popularité ne cesse de prendre de l'ampleur dans les provinces anglophones?

\section{Remerciements}

Cette recherche a été financée par l'Accueil francophone du Manitoba, organisme communautaire chargé de l'accueil, de l'établissement et de l'intégration des immigrants francophones au Manitoba. Elle a été menée avec la collaboration de la DSFM qui nous a facilité l'accès aux comités des parents de ses écoles de Winnipeg.

\section{Références bibliographiques}

Benimmas, A. et Kamano, L. (2009). Comment les futurs enseignants et enseignantes perçoivent-ils l'intégration de l'élève immigrant à l'école francophone en milieu minoritaire au Nouveau-Brunswick. L'établissement international au Canada, 23(2), 6-9.

Benoit, M., Rousseau, C., Ngirumpatse, P. et Lacroix, L. (2008). Relations parents immigrants-écoles dans l'espace montréalais : au-delà des tensions, la rencontre des rêves. Revue des sciences de l'éducation, 34(2), p. 313332. 
Bilodeau, A., Lefebvre, C., Bélanger, J., Deshaies, S. et Gagnon, F. (2010). La collaboration école-famillecommunauté et les mesures qui en sont issues dans quatre communautés montréalaises pluriethniques et défavorisées. Montréal : Centre de santé et de services sociaux de Bordeaux-Cartierville-Saint-Laurent.

Bouchamma, Y. (2008). Les défis de l'école en milieu minoritaire francophone face à l'intégration des élèves immigrants. Canadian Issues/Thèmes canadiens, printemps. (p. 120-123).

Carlson Berg, L. (2010b). Experiences of newcomers to Fransaskois schools: Opportunities for community collaboration. McGill Journal of Education, 45(2),287-304.

Citoyenneté et Immigration Canada. (2015). Canada faits et chiffres : Aperçu de l'immigration résidents permanents et temporaires. Ottawa : Citoyenneté et Immigration Canada. http://www.cic.gc.ca/francais/ressources/statistiques/faits2014/index.asp

Deslandes, R. (2010a). Les conditions essentielles à la réussite des partenariats école-famille communauté. TroisRivières : Centre de transfert pour la réussite éducative du Québec, Université du Québec à Trois-Rivières.

Deslandes, R. (2010b). Le difficile équilibre entre la collaboration et l'adaptation dans les relations école-famille. Dans G. Pronovost, Familles et réussite éducative. Actes de colloque du $10^{\mathrm{e}}$ Symposium québécois de Recherche sur la famille (p. 197-215), Québec : Presses de I’Université du Québec.

Division scolaire franco-manitobaine (DSFM) (2008). Les zones de repêchage des écoles urbaines de DSFM. DSFM. https://www.dsfm.mb.ca/SiteWeb2010/documents/La\%20CSFM/Comit\%C3\%A9s\%20de\%20la\%20CSFM/Co mit\%C3\%A9s\%20ad\%20hoc/Documents\%20utiles/Zones\%20de\%20rep\%C3\%AAchage\%20des\%20\%C3\%A9c oles\%20urbaines\%202008.pdf

Émond, G. (2008). Vivre ensemble dans les écoles de langue française. L'accueil des jeunes immigrantes et immigrants à l'école de la francophonie canadienne. Fédération canadienne des enseignantes et des enseignants.

Emploi et Immigration Manitoba. (2013). Données statistiques sur l'immigration au Manitoba. https://www.gov.mb.ca/labour/immigration/pdf/manitoba_immigration_facts_report_2013.fr.pdf

Farmer, D. (2008). L'immigration francophone en contexte minoritaire : entre la démographie et l'identité. Dans J. Y. Thériault, A. Gilbert et L. Cardinal (dir.), L'espace francophone en milieu minoritaire au Canada (p. 121159). Québec : Fides.

Fournier, V. (2013). Développer la compétence interculturelle. Pédagogie collégiale, 2(26),25-29.

Gagnon, T. (2007). L'école et la diversité culturelle pour l'intégration des familles et enfants immigrants : un défi pour le Nouveau-Brunswick (Mémoire de maîtrise inédit). Université de Moncton.

Gallant, N. et Denis, W. (2008). Relever le défi de la diversité : une comparaison des idéologies en éducation en contexte minoritaire et majoritaire au Nouveau-Brunswick et en Saskatchewan. Éducation et francophonie, 36(1),142-160.

Gerin-Lajoie, D. et Jacquet, M. (2008). Regards croisés sur l’inclusion des minorités en contexte scolaire francophone minoritaire au Canada. Éducation et francophonie, 36(1), 25-43.

Hohlfield, T., Ritzhaupt, A. et Barron, A. (2010). Connecting Schools, Community, and Family with ICT: Four-Year Trends Related to School Level and SES of Public Schools in Florida. Computers and Education, 55, $391-405$.

Jacquet, M., Moore, D. et Sabatier, C. (2008). L'intégration des jeunes immigrants francophones africains dans les écoles francophones en Colombie-Britannique. Vancouver : Vancouver Research on Immigration and Integration in the Metropolis (RIIM) Working Paper Series. http://community.smu.ca/atlantic/documents/Riim-Jacquet_Moore_Sabatier_Masinda2008_000.pdf

Ka, M. (2007). L'immigration francophone au Manitoba, 2000-2006 : un profil statistique. Cahiers franco-canadiens de l'Ouest, 19(2), 119-139.

Alterstice - Revue Internationale de la Recherche Interculturelle, vol. 6, $n^{\circ} 1$ 
Kanouté, F., André, J.V., Charette, J., Lafortune, G., Lavoie, A. et Gosselin-Gagné, J. (2011). Les relations écoleorganisme communautaire en contexte de pluriethnicité et de défavorisation. Revue des sciences de l'éducation de McGill, 46(3).

Kanu, Y. (2009). Parcours scolaires favorisant l'intégration sociale des élèves réfugiés africains au Manitoba. Nos diverses cités, 6 , p. 125-130.

Mackay, T. et Tavares, T. (2005). Building hope: Appropriate programming for adolescents and young adult newcomers of war-affected backgrounds and Manitoba schools (Rapport préliminaire). Manitoba Education, Citizenship and Youth. www.edu.gov.mb.ca/k12/cur/diversity/eal/building_hope.pdf

Malhotrat, N. (2011). Études Marketing (6 éd.). Paris : Pearson Éducation.

Mc Andrew, M., Garnett, B., Ledent, J. et Ungerleider, C. (2008). La réussite scolaire des élèves issus de l'immigration : une question de classe sociale, de langue ou de culture ? Éducation et francophonie, 36(1), 177-196.

Mc Andrew, M, Ledent, L. et Ait-Said, R. (2006). L'école québécoise assure-t-elle l'égalité des chances ? Le cheminement scolaire des jeunes noirs au secondaire. Cahiers québécois de démographie, 35(1), 123-148.

McBrien, J. L. (2005). Discrimination and Academic Motivation in Adolescent Refugee Girls (Thèse de doctorat inédite). Emory University.

Moke Ngala, V. (2005). L'intégration des jeunes des familles immigrantes francophones d'origine Africaine à la vie scolaire dans les écoles secondaires francophones dans un milieu urbain en Alberta: conditions et incidences (Mémoire de maîtrise inédit). Faculté St-Jean, Edmonton.

Murdoch, J et Ledent, J. (2010). Academic performance of youths of immigrant background in Canada: a study from Quebec. http://epc2010.princeton.edu/papers/100317

Piquemal, N. et Bolivar B. (2009). Discontinuités culturelles et linguistiques: portraits d'immigrants francophones en milieu minoritaire. Revue de l'intégration et de la migration internationale, 10(3), 245-264.

Piquemal, N., Bolivar, B. et Bahi, B. (2009). Nouveaux arrivants et enseignement en milieu franco-manitobain : défis et dynamiques. Cahiers franco-canadiens de l'Ouest, 21(1-2), 329-355.

Piquemal, N. et Murenzi, E. (2009). Les enseignants et les nouveaux arrivants : Passion, vocation, défis et stratégies. Revue des Éducatrices et Éducateurs Francophones du Manitoba, 36(1), 10-13.

Robineau, A. (2010). État des lieux sur l'intégration des élèves issus de l'immigration dans les écoles de langue française en situation minoritaire. Moncton : Institut canadien de recherche sur les minorités linguistiques.

Taylor, S. et Bogdan, L. (1984). Introduction to qualitative research methods: the search for meaning. New York : Wiley.

Therrien, J. (2002). L'accueil des personnes immigrantes d'expression française au Manitoba. Winnipeg : Vidacom.

Unesco. (2011). La crise cachée : les conflits armés et l'éducation. Rapport mondial de suivi sur l'EPT. http://www.unesco.org/new/fileadmin/MULTIMEDIA/HQ/ED/pdf/gmr2011-summary-fr.pdf

Vatz Laaroussi, M., Kanouté, F. et Rachédi, L. (2008). Les divers modèles de collaborations familles immigrantesécoles : de l'implication assignée au partenariat. Revue des sciences de l'éducation, 34(2), 291-311. 